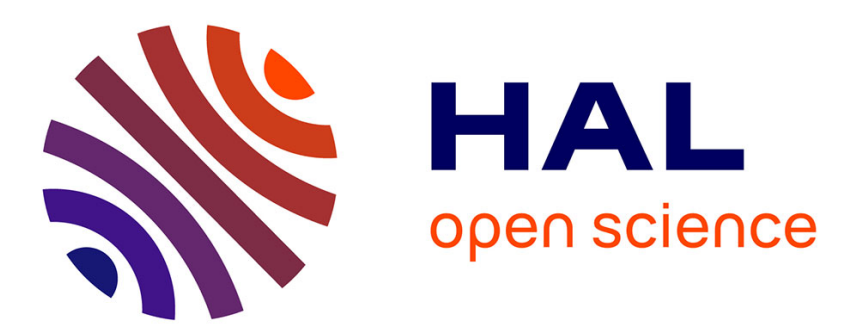

\title{
Les coopérations de proximité dans l'Arc jurassien franco-suisse: Un enjeu de la cohésion sociale transfrontalière
}

\author{
Alexandre Moine
}

\section{To cite this version:}

Alexandre Moine. Les coopérations de proximité dans l'Arc jurassien franco-suisse: Un enjeu de la cohésion sociale transfrontalière. Revue Géographique de l'Est, 2017, vol.57 (1-2 ). hal-01655672

\section{HAL Id: hal-01655672 \\ https://hal.science/hal-01655672}

Submitted on 5 Dec 2017

HAL is a multi-disciplinary open access archive for the deposit and dissemination of scientific research documents, whether they are published or not. The documents may come from teaching and research institutions in France or abroad, or from public or private research centers.
L'archive ouverte pluridisciplinaire HAL, est destinée au dépôt et à la diffusion de documents scientifiques de niveau recherche, publiés ou non, émanant des établissements d'enseignement et de recherche français ou étrangers, des laboratoires publics ou privés. 


\section{Les coopérations de proximité dans l'Arc jurassien franco-suisse : Un enjeu de la cohésion sociale transfrontalière}

Die lokale Zusammenarbeit in dem französisch-schweizerischen Jura : Eine Frage der grenzüberschreitenden sozialen Zusammenhalt

Local cooperation in the Franco-Swiss Jura : An issue of cross-border social cohesion

\section{Alexandre Moine}

revues.org

Édition électronique

URL : http://rge.revues.org/6045

ISSN : 2108-6478

Référence électronique

Alexandre Moine, «Les coopérations de proximité dans l'Arc jurassien franco-suisse : Un enjeu de la cohésion sociale transfrontalière », Revue Géographique de l'Est [En ligne], vol.57 / 1-2 | 2017, mis en ligne le 18 septembre 2017, consulté le 23 novembre 2017. URL : http://rge.revues.org/6045

Ce document a été généré automatiquement le 23 novembre 2017.

Tous droits réservés 


\title{
Les coopérations de proximité dans l'Arc jurassien franco-suisse : Un enjeu de la cohésion sociale transfrontalière
}

\author{
Die lokale Zusammenarbeit in dem französisch-schweizerischen Jura : Eine Frage \\ der grenzüberschreitenden sozialen Zusammenhalt \\ Local cooperation in the Franco-Swiss Jura: An issue of cross-border social \\ cohesion
}

\section{Alexandre Moine}

\section{Introduction}

1 Les territoires transfrontaliers sont soumis à des dynamiques spécifiques qui ont été largement étudiées et interprétées dans le cadre de l'Arc jurassien (Moine, 2012, 2013, 2014 ; Rérat et al. 2011). Ces périphéries sont souvent considérées comme des lieux plus ou moins isolés, qui deviennent des lieux de richesse plus ou moins marginaux (Moullé, 2013), dont le pouvoir décisionnel est réduit (au regard de la complexité des processus qui s'y déroulent) et dépendent de centres nationaux respectifs (Rokkan 1970 ; Shils, 1975). Les périphéries s'inscrivent cependant dans des réseaux complexes où les centres peuvent être/devenir périphéries et vice-versa selon l'échelle géographique (Reynaud 1981 ; Lévy 2003), mais on ne leur reconnaît que rarement le statut de centralités périphériques dotées des moyens requis pour accompagner des logiques socioéconomiques spécifiques. Ainsi, la vision traditionnelle correspond à un espace-frontière considéré comme un territoire marginal et dépendant sur le plan économique et de la vie quotidienne (Paasi, 1996), alors qu'en vérité il dispose d'une certaine autonomie et s'auto- 
organise très largement au travers d'interrelation complexes et de coopérations de proximité.

2 La frontière implique en effet des actions réciproques tout à fait particulières, en ce sens qu'elle se fixe par le truchement des interactions entre deux individus ou groupes, qui limitent leur influence les uns envers les autres en ne voulant pas ou ne pouvant pas agir au-delà de la limite fixée, mais en trouvant des solutions hybrides pour faire ensemble malgré la limite. Selon Georg Simmel (Simmel, 1999) « Si cette notion universelle de limitation réciproque est tirée de la frontière spatiale, celle-ci n'est pourtant, plus profondément, que la cristallisation ou la spatialisation des processus psychiques de délimitation, seuls effectifs. Ce ne sont pas les pays, les terrains, les territoires de villes ou de cantons qui se limitent mutuellement, mais leurs habitants ou propriétaires qui exercent cette action réciproque (...) ». Nous pouvons bien entendu élargir cette vision à l'ensemble des citoyens, groupes qui agissent de part et d'autre et au travers des frontières, mais en nous interrogeant non pas sur des différences de comportements qui sont bien naturelles, mais sur la vision que les uns et les autres ont de la frontière et de leurs voisins frontaliers (Krämer, 2004), modifiant ainsi la portée de celle-ci. Nous postulons alors que des processus d'appropriation, de régulation, de construction sociale et identitaire peuvent amener la pérennité et l'autorenforcement du territoire (Leloup, Moyart, 2015), notamment autour de symboles (Eder Sandtner, 2002), nombreux dans le cadre des coopérations de proximité. Ajoutons enfin qu'une «identité régionale peut être façonnée aussi bien dans un espace transfrontalier que transculturel, si toutefois des rapports transfrontaliers existent " (Raffestin, 1981). C'est ici que nous postulons que la cohésion sociale qui découle des petites coopérations induit un rapprochement entre les populations sous l'angle de la solidarité et d'une conscience collective (Paugam, 2007) autour du développement local transfrontalier.

Nous avons par conséquent lancé une vaste enquête à l'échelle de l'Arc jurassien, afin d'évaluer l'importance de ces coopérations, de comprendre de quelle manière elles naissaient, comment elles se développaient et sur quels moyens elles s'appuyaient. Menée durant un an à cheval sur 2015-2016, cette enquête a permis de mettre au jour plus de 70 coopérations de proximité et d'en interroger plus d'une quarantaine. Les thématiques en jeu sont variées, les formes des coopérations également, tout autant d'ailleurs que les attentes des porteurs de projets dont les moyens sont finalement très réduits alors que les enjeux de cohésions sociale sont immenses... Nous souhaitons donc rendre compte de l'inégale répartition spatiale de ces coopérations, de manières de faire souvent originale et nous pointons les chemins, les attentes et les besoins de ces porteurs de projet souvent discrets et pourtant vecteurs de liens systématiques entre les sociétés françaises et suisses de l'Arc jurassien. L'objectif est finalement de montrer que des coopérations interterritoriales (transfrontalières ici) favorisent la rencontre au point d'imaginer de nouvelles formes de territorialisations communes.

3 Au final, la cartographie est sans équivoque, nous sommes face à un mouvement d'ampleur qui s'incarne à travers les nombreux porteurs de projets, leur localisation, mais également les multiples lieux qu'ils animent de manière continue ou ponctuelle. Ces coopérations se donnent à voir la plupart du temps, rappelant les différences mais également ce qui lie les populations de part et d'autre de la frontière franco-suisse. Ainsi au-delà des coopérations en elles-mêmes, notre étude interroge sur le regard que nous devons porter sur la manière d'envisager la coopération transfrontalière. Si la dimension spatiale des projets est importante, nous notons également l'apport absolument essentiel des multiples relations interpersonnelles qui constituent la base d'un vivre-ensemble 
synonyme de cohésion et de développement plus intégré et plus harmonieux, pour passer du stade des objectifs communs à celui de projet collectif, au sein d'un espace transfrontalier fondé sur des identités mieux partagées (Leresche, Saez, 1997).

\section{Cadre de l'étude}

\section{Développement frontalier, coopération transfrontalière et cohésion}

4 C'est dans cette perspective de comprendre les relations qu'entretiennent les populations de part et d'autre de la frontière franco-suisse dans l'Arc jurassien, et la vision partagée qui en ressort, que nous nous sommes intéressés aux coopérations de proximité qu'elles mettent en place et qui s'y développent. Il apparaît qu'un certain nombre d'entre-elles s'établissent plus ou moins spontanément, sur la base de porteurs français et/ou suisses. De petite taille, visibles ou non aux échelles locales, elles présentent, selon nous, un grand intérêt dans le cadre de la mise en place d'un sentiment communautaire dans l'Arc jurassien, qui repose sur l'expérience de la relation, de la rencontre, de l'échange et du regard croisé. En effet, le devenir de ce espace transfrontalier doit s'ancrer dans le partage des valeurs des deux pays frontaliers afin d'endiguer les risques de repli identitaire ${ }^{1}$ fréquemment évoqués en lien avec le travail frontalier dont la stigmatisation se renforce y compris dans l'Arc jurassien. Rien de tel alors que des rencontres et des manifestations civiques, économiques, sociales et culturelles ${ }^{2}$ dynamiques et mobilisatrices pour entretenir un sentiment d'identité partagée en tenant compte du fait que la coopération devrait se dérouler au niveau territorial le plus élémentaire possible, le plus proche des citoyens, si l'on veut qu'elle soit soutenue par la population vers une identité régionale transfrontalière (Eder Sandtner, 2002).

\section{Une enquête couvrant la totalité de l'Arc jurasssien franco-suisse}

5 L'arc jurassien franco-suisse, massif de moyenne montagne, s'étend sur plus de $200 \mathrm{~km}$ (Carte $n^{\circ} 1$ ). Son relief n'altère pas la circulation des personnes et des marchandises (Moine, 2003) que favorisent par ailleurs les accords de libre circulation et de libre échange signés en 2004 et progressivement mis en œuvre ensuite (Crevoisier et al., 2006). La remise en question récente des accords de libre circulation suite à l'initiative du parti UDC qui souhaite privilégier les emplois à destination des natifs, en février 2014, ouvre une brèche dans le système en équilibre jusqu'alors, fondé sur le développement du travail frontalier en Suisse, le tourisme d'achat en France, les échanges autour du travail à façon entre les entreprises ou encore les mouvements résidentiels de part et d'autre de la frontière (Gertsch et al., 2012 ; Rérat et al., 2011 ; Dubois, 2012). Bien que l'on ne note pas de bouleversements à l'heure actuelle, puisque les conséquences finales de ce vote ne seront connues que trois ans après l'initiative, force est de constater que l'ambiance qui sous-tend le développement transfrontalier de l'Arc jurassien s'est refroidie.

Pourtant, ce territoire de près de 640000 habitants, caractérisé par un archipel de petites villes (entre 4000 et 20000 habitants au plus en France, 5000 et 35000 habitants en Suisse) et qui ne présente pas de centres d'importance (Besançon, Montbéliard, Belfort, Bienne, Neuchâtel, Lausanne se localisent sur les piémonts), ne peut se développer qu'autour de synergies transfrontalières. C'est donc dans ce cadre que nous avons souhaité comprendre comment se mettaient en place des coopérations de proximité qui 
nous paraissent pouvoir entretenir un sentiment communautaire, contre-pied souhaitable aux initiatives qui visent à opposer certaines populations des deux pays. L'étude mise en place se fonde sur une enquête qui s'est déroulée de mai 2015 à avril 2016 dans la totalité de l'Arc jurassien, et a donné lieu à cinq restitutions de terrain (Les Brenets, Porrentruy, Sainte-Croix, Le Sentier et Villers-le-Lac) de manière à confronter les résultats au regard des acteurs politiques locaux et de la société civile.

Carte 1 : Organisation de l'Arc jurassien et périmètre d'enquête

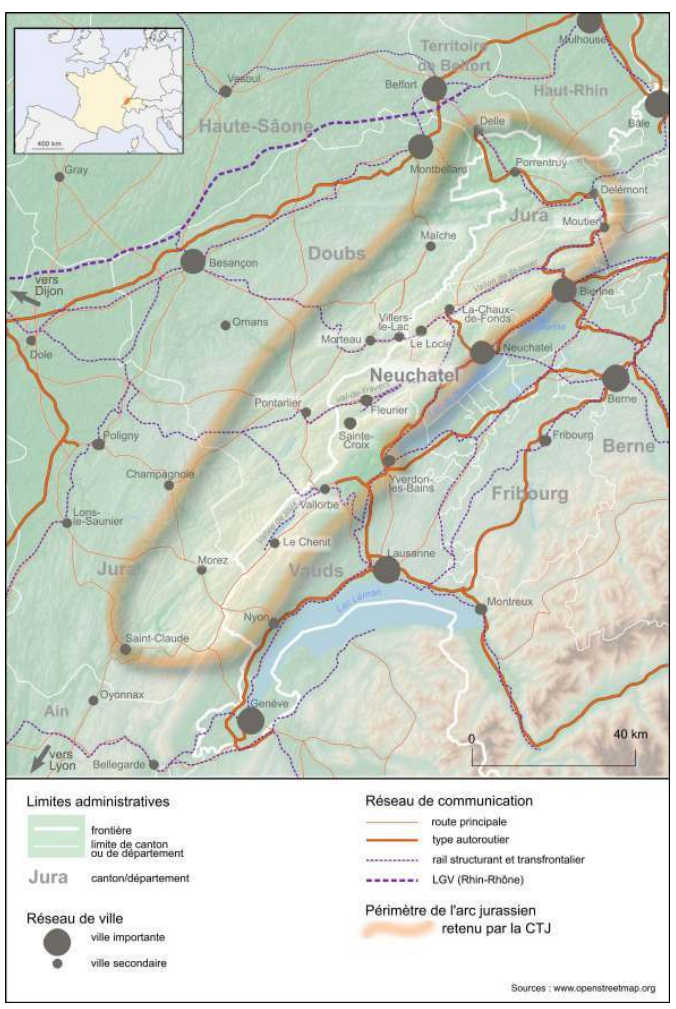

7 L'enquête par questionnaire a dans un premier temps nécessité une recherche de terrain importante, avec l'identification des projets de coopération et de leurs porteurs. En effet, aucun organisme ne recense les coopérations transfrontalières de proximité, lorsqu'elles ne s'inscrivent pas dans le cadre normé des programmes Interreg. Nous avons donc procédé de quatre manières :

Identifier au sein du programme Interreg IV (2007-2013) les coopérations qui correspondaient à nos critères, c'est-à-dire des projets de proximité qui impliquent des échanges entre acteurs suisses et français de la société civile et/ou de collectivités locales et se traduisent par des réalisations communes concrètes, visibles et dont les effets sur la mise en lien des populations sont durables dans le temps. Sur 61 projets financés, 7 entrent dans nos critères, ils demeurent opérationnels après l'extinction des aides et ne concernent pas des études ou réalisations ponctuelles liées à la durée de l'aide. S'ajoutent deux projets ayant été soutenus respectivement par Interreg II et Interreg III, et qui demeurent opérationnels à ce jour ;

Recourir à la presse quotidienne régionale $(\mathrm{PQR})$ s'est révélé décisif. Ce support a très bien été documenté méthodologiquement (Le Lay, Rivière-Honnegger, 2009). La PQR tient la seconde place au sein des media français en termes de diffusion, et nous nous sommes appuyé sur un ensemble de titres français (L'Est républicain qui couvre l'ensemble du 
Haut-Doubs, et le Progrès pour le Haut-Jura) et suisses (La Tribune, L'impartial, etc.) pour repérer des évènements franco-suisse qui reposent sur des coopérations de proximité ;

Effectuer des recherches sur internet avec des mots-clés adaptés ont permis de repérer quelques coopérations supplémentaires ;

Enfin, le bouche à oreille autour de notre enquête, les multiples contacts avec des porteurs de projet et/ou des acteurs de la coopération ont été déterminants.

8 Au final nous avons comptabilisé 73 projets dont 41 ont fait l'objet d'une enquête par entretien semi-directif d'une à deux heures. Si l'échantillon enquêté est représentatif par rapport à la totalité des projets recensés, notons malgré tout qu'au regard de la population totale de l'Arc jurassien les résultats sont à observer avec prudence, les petites coopérations transfrontalières mobilisant de fait un nombre réduit de porteurs.

9 Nous avons ainsi interrogé les porteurs de projet français et suisses sur quatre points saillants que nous avions auparavant identifiés : 1.) Les caractéristiques du projet enquêté (thématique, localisation, type de la structure, taille, date de création, etc.) ; 2.) De quelle manière leur projet a émergé (depuis quand, pourquoi, objectifs, description de la coopération, financement, etc.) ; 3.) Les conditions dans lesquelles se déroulait la coopération (apport de la coopération, coût obstacles, etc.) ; et 4.) La pérennisation de la coopération (aides, outils, problèmes).

10 Les projets identifiés ainsi que leurs porteurs, ont été spatialisés au sein des quatre aires de coopération reconnue par les différents partenaires de la coopération (CTJ, 2016). Par ailleurs nous avons, lorsque cela est judicieux, tiré un parallèle entre le profil de nos petits projets de coopération et celui des projets Interreg 2007-2015. En effet au fur et à mesure de nos investigations, une hypothèse s'est fait jour: les petits projets de coopération semblent s'inscrire en complémentarité de la coopération Interreg, tant d'un point de vue des thématiques abordées que de la localisation des projets et de leurs porteurs ou encore de la taille des financements... A ce titre notre méthodologie fondée sur une enquête de terrain, se différencie tout en les complétant, des démarches engagées dans les différents programmes européens ESPON à l'échelle européenne, qui visent à caractériser les espaces transfrontaliers et à comprendre les coopérations institutionnelles soutenues dans le cadre de programmes européens ${ }^{3}$.

\section{Le profil de la petite coopération}

\section{La culture domine les petites coopérations}

11 Sur l'ensemble des 73 projets recensés lors de notre enquête, près de $55 \%$ sont adossés à la culture, auxquels il faut ajouter $24 \%$ adossés au tourisme... ces deux thématiques entretenant d'étroites relations. Si on ajoute les projets d'ordre sportif, également souvent vecteurs de développement touristique, soit $8 \%$ des projets, on couvre thématiquement près de $89 \%$ des projets de coopération de proximité (Figure 1). A titre de comparaison, culture, tourisme et sport ne représentent qu'à peine $20 \%$ des projets Interreg 2007-2013. A l'inverse, formation, développement économique, aménagement du territoire, coopération et environnement ne représentent que $11 \%$ des petits projets de coopération recensés, contre $80 \%$ des projets Interreg. 
Figure 1 : Répartition thématique des projets de coopération de proximité (\%)

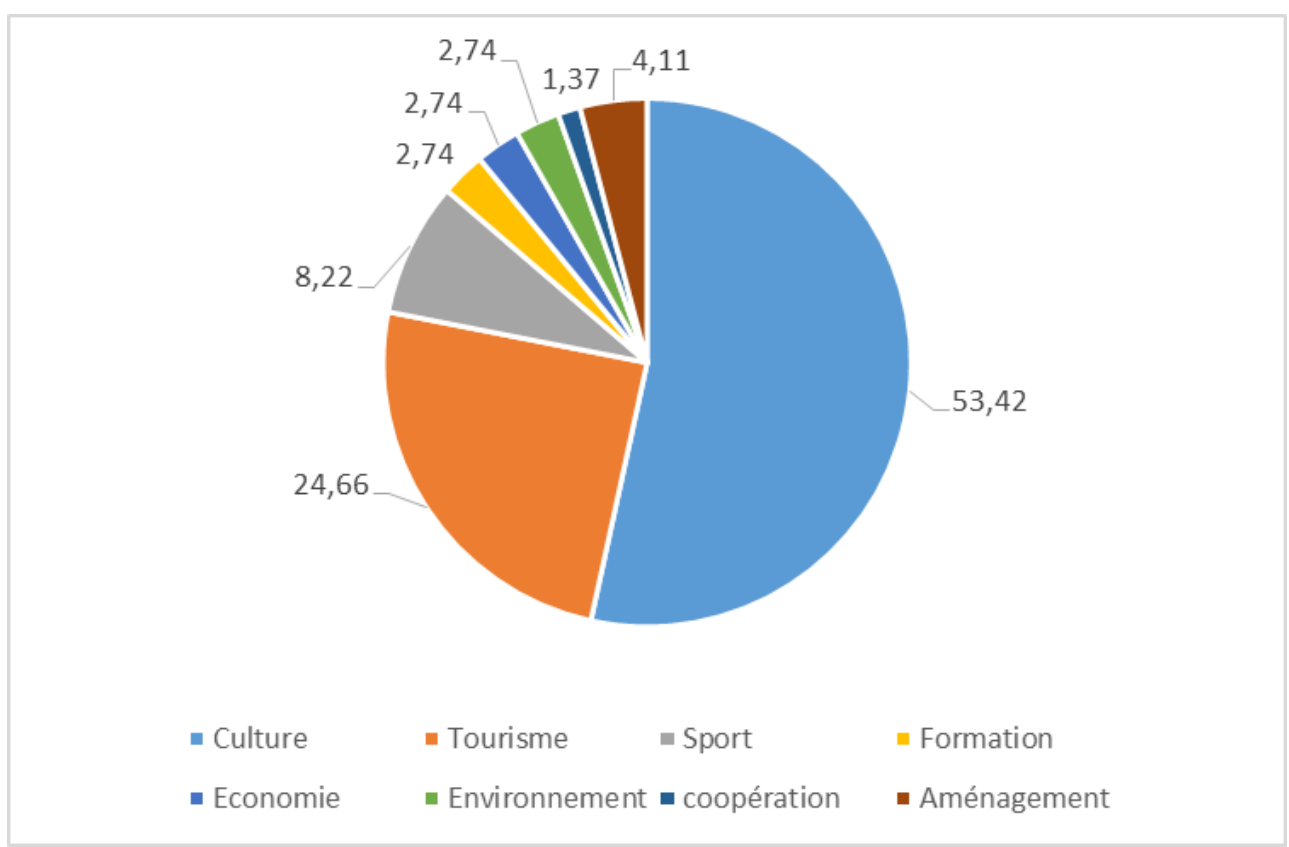

En désagrégeant la thématique culture, nous découvrons une très grande diversité de coopération: littérature, théâtre, musique sous de nombreuses formes, archéologie, danse, photographie, beaux-arts, arts du cirque, ski de fond, randonnée, etc. Ce foisonnement est remarquable et soutient l'idée que la coopération transfrontalière s'immisce dans tous les compartiments de la vie quotidienne, soutenant un développement fondé sur une offre assez riche. La forme de ces coopérations est également très diversifiée : concerts (Lucelle sonore, Impétus, Mômes en scène, Brass Band, orchestre symphonique, jardins musicaux, etc.), spectacles incluant les habitants (Nuits d'été de Milandre), lectures itinérantes (Saute-frontière), fêtes (du Doubs, du Froid, des Bergers, etc.), multiples itinéraires de découverte transfrontaliers (sentier des bornes, mur aux fleurs de Lys, kilomètre zéro, Chemin de la Vy aux moines, Parcours Bourbaki, Sentier des contrebandiers, chemin de la contrebande, chemin des rencontres, etc.), sites en réseau (route de l'Absinthe, route du sel, etc.), courses (ski de fond, VTT, course à pied, etc.), journées d'étude (historiques, archéologiques, etc.)... Cette grande diversité, et la forme même des coopérations interroge la question de leur visibilité.

\section{Des coopérations plus ou moins visibles et mises en valeur}

13 Nous postulons que la visibilité des projets de coopération dans l'espace et leur plus ou moins grande permanence, peuvent impacter le sentiment d'appartenance à un espace transfrontalier commun. A priori, en multipliant les contacts, on multiplie les occasions de mesurer les différences et les ressemblances, de se connaître et se reconnaître dans une destinée commune. En faisant régulièrement des références au pays voisin au travers d'itinéraires transfrontaliers, de spectacles itinérants, en créant des moments festifs partagés, en participant à des aménagements conjoints, sont créées les conditions de l'échange, de la mesure des spécificités du voisin. On peut alors parler d'utilité sociale, à l'instar des réflexions en économie sociale et solidaire, lorsque "l'activité d'une organisation [...] a pour résultat constatable [...] de contribuer à la cohésion, à la solidarité (lien 
social de proximité), à la sociabilité, et à l'amélioration des conditions collectives de développement humain " (Gadrey, 2004). Il nous est donc apparu primordial de renseigner la visibilité des projets et la fréquence de cette visibilité dans le temps. A noter que nous n'analysons pas ici la fréquence des contacts entre personnes, en lien avec le portage des différents projets, cette donnée est difficile à quantifier et à analyser. D'une manière générale, nous retenons tout de même que la plupart des portages induisent des rencontres régulières durant l'année, qui sont autant d'occasions de partager des cultures différentes, autour de la préparation d'un spectacle ou d'un évènement festif, de la mise en place d'un itinéraire transfrontalier, etc. Nos interlocuteurs relèvent alors l'enrichissement mutuel... Pour en revenir à la visibilité des actions de coopération, nous pouvons noter que seulement $22 \%$ des projets ne donnent pas lieu une manifestation ouverte au grand public, au moins une fois par an, sinon $37 \%$ reposent sur une manifestation publique à caractère transfrontalier (par sa programmation, sa localisation), $20 \%$ sont visibles plusieurs fois par an (spectacle itinérants, programmation culturelle croisée) et enfin $20 \%$ s'affichent en permanence (sites d'information, itinéraires transfrontaliers (Figure 2).

Figure 2 : Visibilité des projets de coopération (\%)

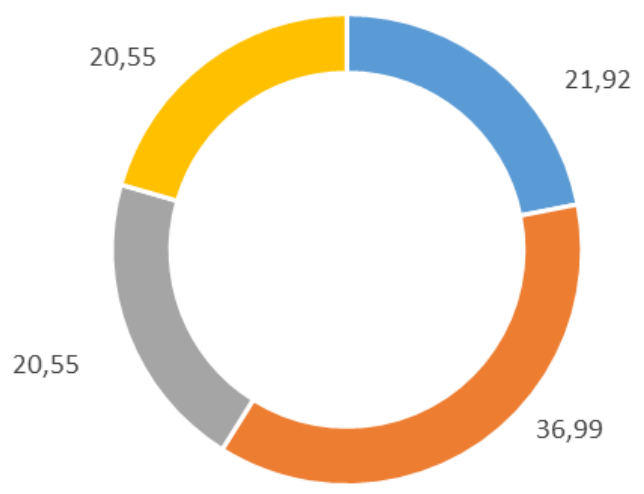

- Invisible - Une fois = Plusieurs fois par an - Permanente

14 Au final, ce sont des centaines de contacts qui s'établissent durant toute l'année entre partenaires suisses et français (Carte $n^{\circ} 2$ ), et assurément des milliers de personnes qui se rencontrent au travers d'éléments de programmation, renforçant le sentiment d'appartenir à un territoire commun qui fait sens. 
Carte 2 : Spatialisation et visibilité des projets de coopération de proximité

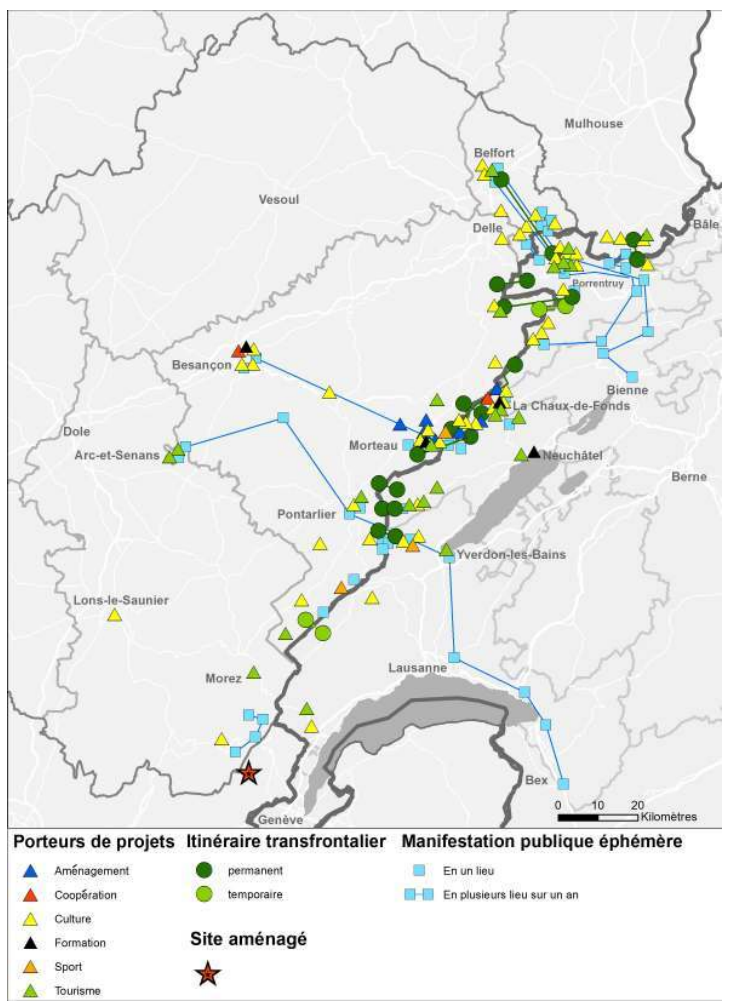

\section{Un net gradient Nord/Sud qui s'explique}

15 Au-delà du volume des projets recensés, nous nous sommes interrogés sur leur répartition spatiale. Loin d'être homogène, celle-ci interroge si on considère le gradient Nord/Sud qui apparait dans l'Arc jurassien. Organisé en quatre aires de coopération successives du Nord au Sud, l'Arc jurassien, s'il présente les mêmes caractéristiques physiques, est par contre diversement intégré en matière de coopération transfrontalière (CTJ, 2016). Il existe quatre territoires aux problématiques et aux enjeux différenciés, qui se sont progressivement structurés depuis plus de 25 ans : au Nord, l'ensemble DellePorrentruy est dynamique, il s'ancre aux piémonts respectifs (Belfort et Bienne) qui le dynamisent. Plus au Sud, l'ensemble liant le Val de Morteau aux villes du Locle et de La Chaux-de-Fonds est de loin le plus intégré d'un point de vue transfrontalier. A elles deux, ces aires de coopérations concentrent près de $70 \%$ des projets recensés dans une parfaire parité ( 25 projets chacune). Plus au Sud encore l'ensemble Mont d'Or-Chasseron concentre $20 \%$ des projets (14) puis l'ensemble Haut-Jura, seulement $10 \%$ (9 projets) (Figure 3). 
Figure 3 : Répartition des projets entre les territoires de coopération

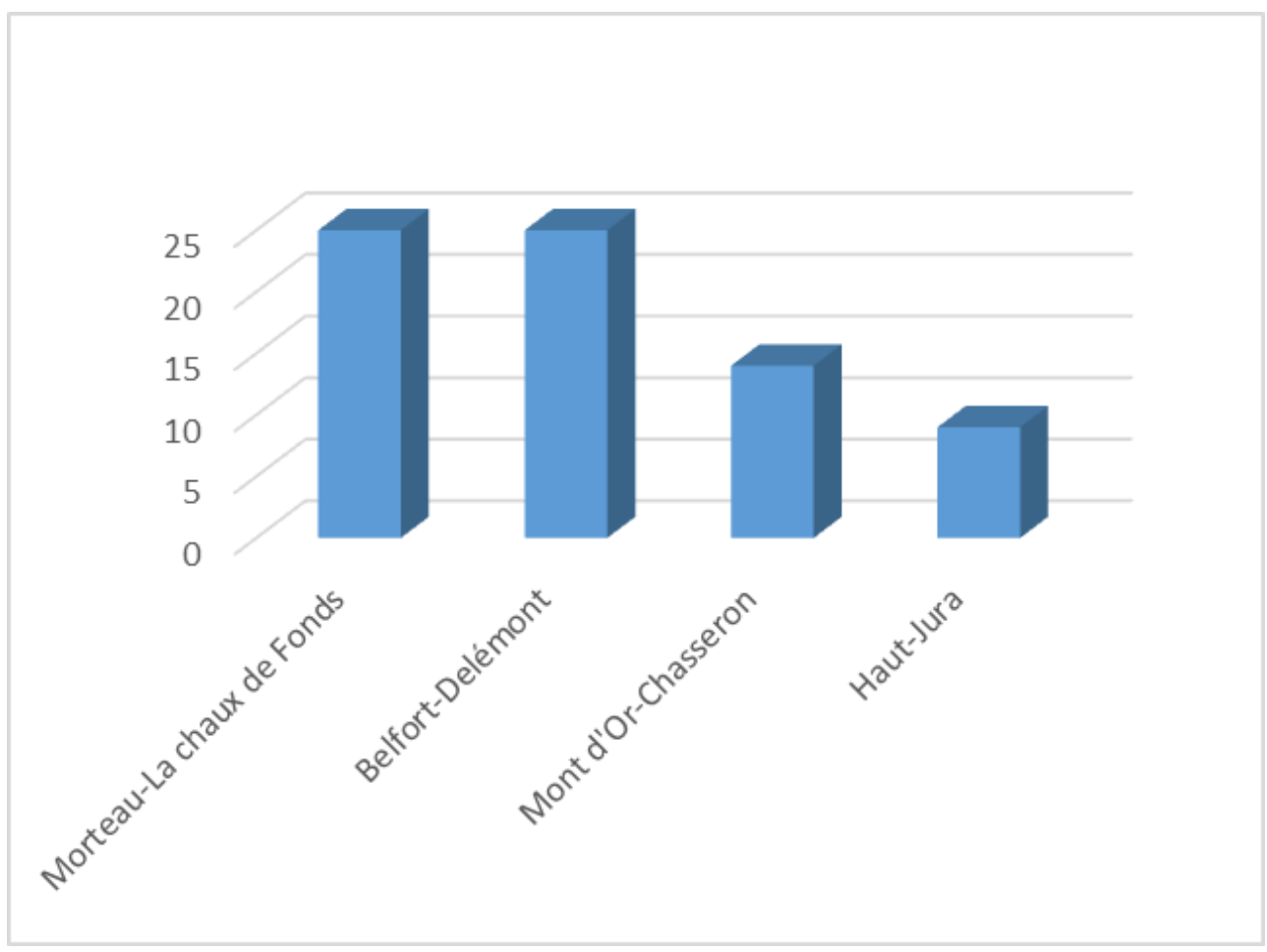

Ces différences ne s'expliquent pas de manière univoque et c'est un ensemble de facteurs croisés qu'il faut convoquer pour comprendre cette situation :

La densité de population tout d'abord, peut suggérer en proportion une occurrence plus importante de contacts entre les habitants. Si nous considérons l'Arc jurassien francosuisse, on repère un gradient Nord/Sud assez prononcé. Une nuance, l'aire BelfortDelémont est nettement plus peuplée que celle de Morteau - La Chaux-de-Fonds alors qu'elles font jeu égal en termes de volume de coopération. De la même manière si Morteau - La Chaux-de-Fonds est globalement aussi peuplée que l'aire Mont d'Or Chasseron, alors que le nombre de coopérations y est quasiment le double ;

La topographie et les difficultés de déplacement d'un pays à l'autre peuvent peser sur la mise en contact des populations. Nous retrouvons effectivement un gradient d'altitude du Nord au Sud de l'Arc jurassien, auquel s'ajoute des conditions climatiques plus ou moins propices aux déplacements en hiver ;

Le sentiment d'isolement et l'enclavement par rapport au pays de référence viennent compléter ces facteurs. En réaction, on note que les porteurs de projets s'ouvrent alors vers le pays voisin lorsqu'ils se considèrent dans une situation de "Finistère ». Ceci est souvent évoqué dans l'aire de coopération Belfort - Delémont, pour rappel le Canton de Jura prend son autonomie vis-à-vis du Canton de Berne en 1979, dès lors se noue le sentiment d'être une exception dans le paysage suisse et l'ouverture vers la France voisine, son important bassin de population, ses nombreux services et ses réseaux à grande vitesse se renforce. Ceci est également vrai à une échelle locale, au sein de l'aire Mont d'Or - Chasseron à l'échelle du plateau des Fourgs $(\mathrm{F})$ vers Sainte-Croix $(\mathrm{CH})$, où à près de 1000 mètres d'altitude dans un secteur enclavé, on recense $35 \%$ de l'ensemble des coopérations de l'aire complète. Ceci est moins vrai pour le Sud du Massif où les deux parties française et suisse s'organisent vers leurs centres respectifs situés en contrebas ; 
Le portage politique est un déterminant supplémentaire, comme nous le verrons plus tard, certaines coopérations ont été initiées par des acteurs politiques, et on note un engagement particulier du Canton de Jura pour la promotion de la coopération transfrontalière avec la France. Même remarque autour de Morteau - La Chaux-de-Fonds où existe le seule Groupement Local de Coopération Transfrontalière (GLCT) de l'Arc jurassien ;

Enfin, le degré d'intégration (Fourny, 2003 ; Sohn, 2009) transfrontalier est prépondérant. Nous entendons par là l'imbrication fonctionnelle et politique portant à la fois sur l'histoire locale, les projets d'aménagement, les multiples flux qui existent, les habitudes des habitants, etc. Ainsi une étude montre que de nombreux frontaliers de Morteau ou Villers-le-Lac interrogés, pratiquent des loisirs en Suisse, y consomment ou y ont développé des réseaux sociaux (Dubois, 2012). Nos analyses successives (Moine, 2015) montrent ainsi que les deux aires où l'on comptabilise le plus de coopération, sont les deux aires les plus intégrées de l'Arc jurassien.

\section{Une dynamique relativement récente}

Si l'on considère maintenant l'historique de ces coopérations, nous constatons que les dates de mises en place ne s'étalent pas de manière homogène dans le temps. Sur le temps long, la série montre un ensemble de créations antérieur aux années 2000 (15\% des projets recensés), suivi d'un creux remarquable jusqu'au milieu des années 2000 suivi d'une reprise assez conséquente à partir de 2007 et un renforcement continu (Figure 4). On peut globalement dire que le mouvement est récent puisque finalement $72 \%$ des projets sont postérieurs à 2007. Peut-il exister un phénomène de cycle, des projets de coopérations s'étant éteints il y a quelques années, que nous ne pourrions pas prendre en compte sauf à faire des recherches dans les archives de la Presse Quotidienne Régionale ? Nous ne pouvons le dire, mais les acteurs interrogés ne mentionnent jamais de telles disparitions, il semble bien que le mouvement soit récent.

Figure 4 : Date de création des coopérations recensées

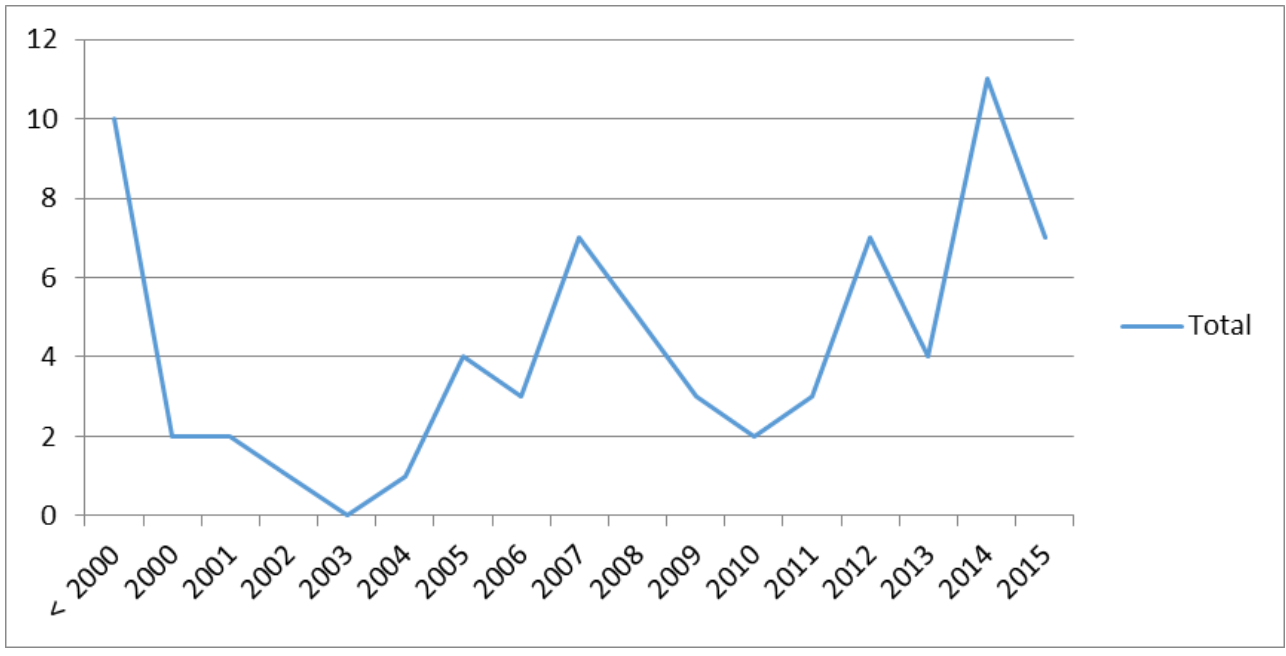

Enfin, si nous reprenons une analyse spatiale de la distribution des créations de projets dans le temps, en fonction des aires de coopération, nous observons deux faits majeurs (Figure 5) : 
Les plus anciennes coopérations se situent entre Morteau et La Chaux-de-Fonds, secteur le plus intégré, mais également dans le Haut-Jura ;

Les créations les plus récentes se font essentiellement dans les aires situées au Nord du Massif et particulièrement à partir de 2006-2007 pour l'aire Belfort - Delémont résultat d'une politique publique en lien avec le service de la coopération du Canton de Jura à cette époque. L'année 2006 constitue également le début d'une dynamique assez soutenue pour l'aire Morteau - La Chaux-de-Fonds sans que nous puissions véritablement l'expliquer...

Figure 5 : Date de création des coopérations recensées par aire de coopération

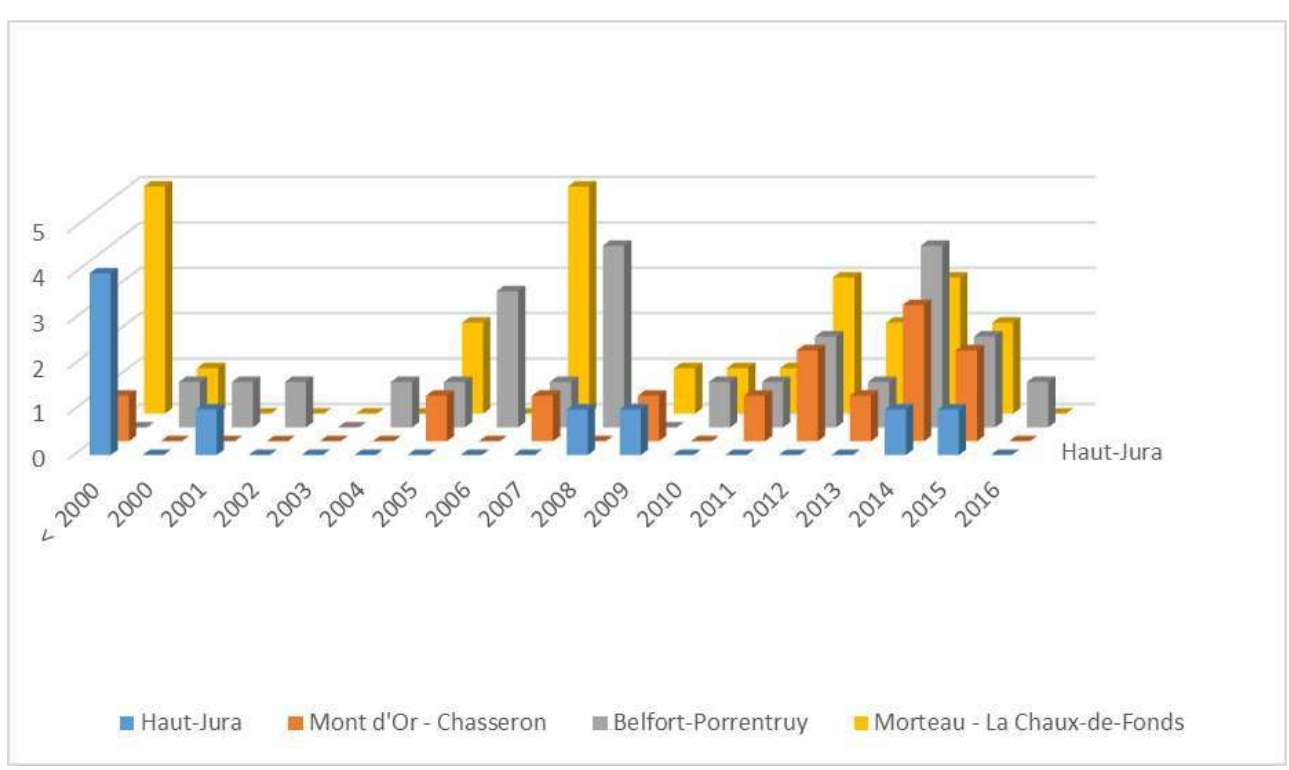

Pour conclure cette première phase d'analyse, nous pouvons dire que le mouvement est d'ampleur. Si l'on cumule notre recensement (73 projets) et le bilan des coopérations Interreg IV (54 projets) ${ }^{4}$, ce sont 127 projets qui animent plus ou moins l'Arc jurassien sur les dix dernières années. L'apparition des petits projets recensé est plutôt récente et semble liée principalement au degré d'intégration des territoires concernés, ainsi qu'à l'importance du portage politique, les deux phénomènes étant souvent liés. Si l'on considère les deux familles de projets, et que nous localisons les porteurs, nous sommes frappés par la dichotomie qui apparaît. Les porteurs de projets Interreg sont essentiellement localisés dans les piémonts du Massif (carte 3), nous pouvons ainsi observer que $75 \%$ des porteurs de projets français sont hors du périmètre de l'Arc jurassien retenu par la CTJ (carte 1), 30 \% des porteurs suisse dont une partie à Neuchâtel sont considérés dans le Massif. A l'inverse, les porteurs de petits projets recensés sont quant à eux situé dans le périmètre de coopération de l'Arc jurassien pour $86 \%$ d'entre eux, témoignant de la proximité entre ceux-ci, les publics et les lieux où se déroulent les projets. Il s'agit là d'une information qui vient appuyer l'importance de leur existence dans la perspective de rencontre des populations et de cohésion transfrontalière à l'échelle du Massif. 
Carte 3 : Localisation des porteurs de projet Interreg IV (2007-2013)

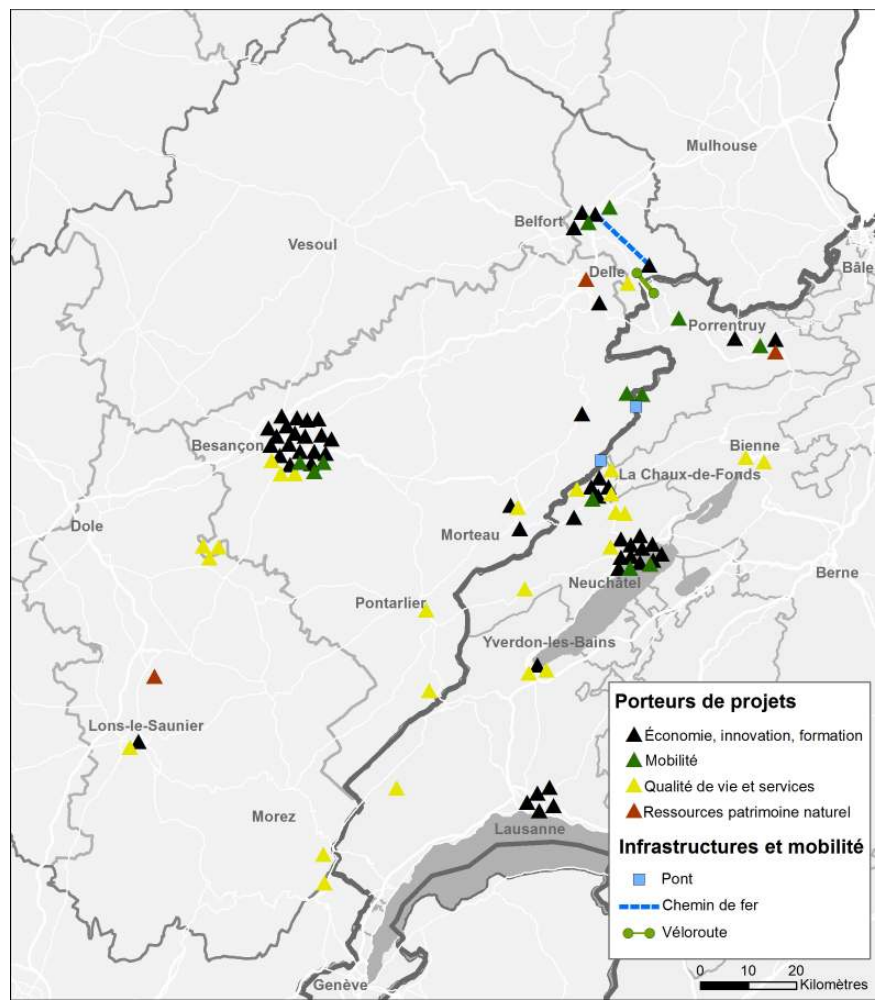

Il s'agit maintenant de s'interroger sur la manière dont ces coopérations ont émergé, comment elles vivent et quelles sont les conditions de leur maintien.

\section{Comment naissent les coopérations ?}

21 Comprendre de quelle manière les petites coopérations fonctionnent est essentiel pour mesurer leur impact en termes de cohésion sociale. Nous avons interrogé plus de 40 porteurs de projets afin de comprendre d'où venait l'envie de coopérer, comment elle se concrétisait par des actions et à quel prix ; comment ensuite ces projets de coopération pouvaient s'inscrire dans la durée. Car finalement, ce que nous observons ne peut avoir de l'effet que dans la répétition et la multiplication des contacts entre citoyens français et suisses.

\section{Les petites coopérations dominées par l'envie...}

Envie et opportunité sont souvent mêlées, lorsque les porteurs de projets évoquent la mise en œuvre de leur coopération, l'une déclenchant l'autre pour près des $2 / 3$ des projets enquêtés. L'opportunité entraîne l'envie d'aller plus loin dans la connaissance de l'autre, alors que l'envie à son tour permet de dessiner des opportunités. Ainsi la coopération semble assez naturelle parce que nous sommes différents mais engagés sur des projets analogues (Lions Club, Rotary Club, Syndicats, Terra Salina, Route de l'Absinthe, Chorales d'Ajoie, Cercle d'Archéologie, etc.), parce que nous partageons des passions identiques (Porrentruy-Delle-Jazz, Brass Band, harmonies, Franco-suisse de pêche, Mara ski de fond, Transjurassienne, Murs et murgers, etc.). La coopération est 
alors support de développement, avec la création d'itinéraires, la réfection de bâti ancien, la rencontre tout simplement, et le passage de frontière est alors enrichissement... Mais si l'envie est absolument indispensable, il apparaît aussi le sentiment de pallier l'absence de résultats de la coopération «officielle ». A ce titre, près de $70 \%$ des projets sont associatifs, contre seulement $18 \%$ portés par des institutions (département, commune), $7 \%$ par des fondations suisses et enfin $5 \%$ par des acteurs privés. Les porteurs de " petites coopérations » de terrain ont ainsi le sentiment de participer à un réel apport à la « grande coopération ».

\section{... et par un ensemble de raisons pragmatiques}

Au-delà de l'envie, un certain nombre de raisons pragmatiques se font jour. La coopération est alors impulsée par le politique (Réseaux de centres culturels, Fest'Hiver, Centre jurassien des Arts de la Scène, Franco-Vélo-Suisse, Les Nuits de Milandre, Terra Salina, Aire Urbaine du Doubs, Chemins de la contrebande, Pôle franco-suisse de sensibilisation et d'éducation à l'environnement, etc.) qui est simplement porteur d'idées, ou soutien financier sans assumer la mise en œuvre matérielle.

La coopération peut également s'inscrire dans le cadre d'une posture, c'est le cas pour certaines collaborations culturelles qui naturellement s'étendent au-delà de la frontière. Dans certains cas on parlera tout naturellement de bassin de vie franco-suisse (Aire urbaine du Doubs, Forum transfrontalier, concert symphonique au cœur des pâturages, festival du terroir sans frontière, Evidanse, etc.).

On observe également que la frontière est vécue comme une ressource (Sohn, 2014), la coopération sert alors à élargir les collaborations, avec un but touristique affirmé, sur la base de projets culturels. La frontière attise alors l'intérêt, suscite des rencontres, étonne, rassemble aussi et pose l'exotisme du voisin comme source d'attrait. Il s'agit alors de faire circuler les personnes, d'élargir une aire de chalandise et une offre culturelle (Evidanse, Fest'Hiver, Echanges culturels de l'AICC, Lucelle sonore, Ex-Situ, Réseau TRIPTIC, Terra Salina, Jardins musicaux, Route de l'absinthe, etc.), ou encore de rapprocher autour de la patrimonialisation (programmation muséale sur la grande Guerre, chemin des bornes, $\mathrm{km}$ 0) où la frontière objet du passé, conduit à l'unification dans une vision progressiste (Fourny, 2003);

La coopération est également un support de développement, dans une région de montagne enclavée, lorsque artisans et commerçants se retrouvent autour d'un marché local transfrontalier. Si la frontière a tendance à isoler, elle se pose aussi comme une ouverture vers un autre système, politique, institutionnel, économique qui offre des opportunités (Evidanse, Aire urbaine du Doubs, Impétus, Festival des terroirs sans frontière, etc.) ;

Enfin, si la coopération est le plus souvent entreprise par des initiatives de terrain, elles émergent parfois en phase avec de grands projets extérieurs (la Franco vélo suisse, étape d'un circuit transeuropéen La Rochelle-Francfort). 


\section{Comment fonctionnent les petites coopérations?}

\section{De petits moyens pour des résultats de grande envergure}

Dans un premier temps la coopération ne nécessite pas des moyens importants. Les envies, les idées sont là, quelques fois le starter est politique, dans tous les cas les premiers pas coûtent peu. Passés le temps de la prise de connaissance, s'engage le temps de la collaboration. Les rencontres se multiplient, il faut identifier des contacts, comprendre les différences, adapter les manières de voir et de faire... et cela prend du temps. S'il est relevé que coopérer ne coûte finalement pas plus que de se développer seul, il semble tout de même qu'un accompagnement de la coopération est nécessaire. Il est en effet mentionné que le surcroît d'énergie que demande la coopération, appelle des contreparties financières, certes modestes, mais nécessaire pour maintenir l'envie, l'attention, au risque sinon de voir s'installer une certaine lassitude. Lorsque le projet prend corps, l'envie d'aller plus loin engage dans certains cas des coûts qui, s'ils ne sont pas considérés à leur juste valeur, peuvent obérer la poursuite de la coopération. Ceci étant, au final, ce sont plusieurs dizaines de milliers de personnes ${ }^{5}$ qui partagent d'une manière ou d'une autre un sentiment autour de la reconnaissance des acteurs et habitants du pays voisin.

\section{La nécessité de pérenniser la coopération par les financements}

La recherche de financement paraît compliquée... Suivant si le projet est porté par un acteur français ou suisse ou par l'un et l'autre simultanément, l'identification de partenaires financiers ou d'aides est difficile. Si la France et sa complexité institutionnelle fondée sur des compétences croisées et de nombreux partenaires laissent souvent perplexes les porteurs de projets, la Suisse au contraire est saluée pour la simplicité des démarches et la présence de fondations qui accompagnent volontiers les projets culturels qui rappelons-le constituent le socle des coopérations de proximité. Ainsi certains projets sont délibérément appuyés sur une association suisse afin de mobiliser plus facilement ces financements privés. Nous n'avons pas engagé d'analyse précise des budgets des projets étudiés, néanmoins nous pouvons affirmer que la contribution des partenaires suisses aux coopérations de proximité est largement plus forte que celle des partenaires français, qu'ils soient institutionnels ou privés. Ainsi, plus de la moitié des projets (55\%) sont portés par un seul partenaire, plus volontiers suisse ( $35 \%)$ que français $(20 \%)$, sinon par deux partenaires français et suisse ou plus de deux... et appuyés sur des structures distinctes (Figure 6). 


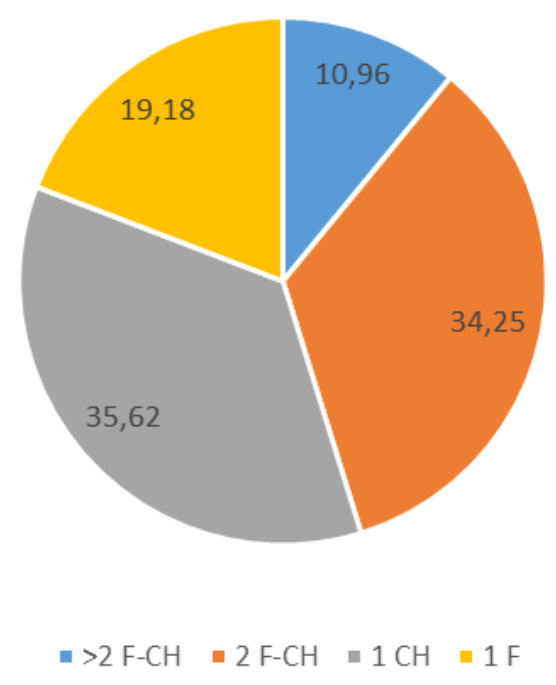

\section{Le développement transfrontalier n'est pas une priorité}

30 Il est rapporté fréquemment que l'accès aux informations portant sur des partenaires potentiels est déficiente. Les recherches, même si elles sont facilitées par l'usage d'internet, peuvent être longues. Par ailleurs, il n'existe pas de dispositif à l'échelle de l'Arc jurassien ou de ses quatre aires de proximité, qui permette d'identifier et de connaître les projets de coopération existants. Ce manque de lisibilité de l'existant handicape les acteurs potentiellement porteurs de projets de coopération.

31 Ensuite, les coopérations de proximité se développent au gré des porteurs, dans certains cas les relations nouées sont intenses, les contacts fréquents, alors que d'en d'autres cas la dynamique est plus ténue. Une chose est cependant certaine, il n'existe quasiment pas de dispositifs transfrontaliers à part le GLCT AUD et le Forum Transfrontalier ${ }^{6}$, ce qui signifie que le développement transfrontalier n'est pas un objectif pour les porteurs de petites coopérations, mais qu'il découle d'activités dont l'extension transfrontalière est postérieure à la mise en place initiale de l'activité. Ce constat nous ramène à l'importance de la mise en œuvre d'actions transfrontalières de fond afin de systématiser l'ouverture vers le pays voisin.

\section{Du besoin d'outiller les petites coopérations}

Le profil des porteurs des petites coopérations est une indication importante pour expliquer les besoins qui se font jour. Comme nous l'avons mentionné, ces coopérations reposent essentiellement sur les mouvements associatifs, avec leur corollaire de petits moyens, d'engagements très personnels, d'épuisement lorsque la reconnaissance n'est pas au rendez-vous. Si elles sont discrètes, les petites coopérations exigent cependant une attention qui à ce jour fait défaut dans la plupart des cas que nous avons rencontrés.... Les attentes sont pourtant assez simples : 
Les petites coopérations sont nombreuses, elles montrent un dynamisme certain et les porteurs de projets sont unanimes pour dire que la dimension transfrontalière est une plus-value certaine. Mais, même si ces coopérations se développent avec peu de moyens, il apparaît indispensable de les soutenir financièrement. Alors qu'Interreg est aujourd'hui dédié aux projets importants, il est indispensable qu'un fond simplifié porté par les instances de la coopération (Conférence Transjurassienne et Interreg) soit dédié aux coopérations de proximité, c'est ici que se joue la cohésion territoriale transfrontalière ;

Quatre fils conducteurs se font jour dans l'Arc jurassien : l'importance de l'horlogerie comme un socle identitaire local, les réseaux pédestres et VTT qui structurent les territoires traversés en effaçant la frontière, le Doubs comme un ensemble de lieux sur la frontière qu'il dessine, et enfin le massif par le cadre et les conditions de vie qu'il impose. Il s'agit de s'appuyer sur ces «totems » afin de multiplier les occasions de coopérer et de renforcer l'économie présentielle (Segessemann, 2015) en multipliant les liens et les lieux de contact notamment dans une perspective de développement touristique ;

La société civile joue un rôle important dans les interstices, c'est là que se situe le ciment du vivre-ensemble, au travers de multiples petits actes qui prennent un sens plus global lorsqu'on les analyse à l'échelle de l'Arc jurassien. Néanmoins, à l'échelle locale, il est relevé l'importance du «bricolage », des contournements, de la « contrebande », pour surmonter les tracasseries administratives, les blocages dus à la frontière... Les coopérations de proximité sous-entendent des déplacements de personnes et de matériels sur de courtes distances qu'il s'agit de faciliter au maximum en levant temporairement les obligations douanières autour de couloirs francs.

Ces coopérations doivent être connues, documentées et observées afin d'assurer leur pérennité, leur visibilité et l'émergence de nouveaux projets. Il s'agit donc mettre en place une veille qui permette l'actualisation permanente des données décrivant les coopérations de proximité, et soutienne une véritable intelligence collective autour de la coopération.

\section{Conclusion}

$\mathrm{Au}$ sortir d'un an d'enquête, nous sommes en mesure de dire qu'un important mouvement de coopération contribue au rapprochement de nos populations frontalières, qu'il est source de cohésion sociale, de bien vivre ensemble et pourquoi pas d'identité partagée. Alors que les tensions entre les personnes se font plus vives en ces périodes de ralentissement économique, il est plus urgent que jamais de reconnaître ces multiples porteurs de projets et de les accompagner dans leur entreprise. Rappelons que c'est avec de faibles moyens que ces coopérations se développent et renforcent la cohésion de l'espace transfrontalier, et sans relais efficaces, constants et engagés, ni reconnaissance, les résultats demeureront confidentiels et sans impact notable sur le développement transfrontalier et le «bien vivre ensemble » des populations de l'Arc jurassien. Pourtant, il nous semble possible d'atteindre certains objectifs, mais cela nécessite un relais institutionnel, même si la force de ces dynamiques est d'être en dehors de toutes structures. Il convient de les accompagner financièrement afin de leur permettre de maintenir leur engagement. Le temps est en effet venu d'accorder plus de moyens à ceux qui font la coopération, qui s'engagent sur des projets qui fédèrent plus qu'ils ne divisent, au risque sinon de voir les énergies s'éteindre. Force est de constater cependant, une forte inertie, au sein d'un territoire où les habitudes politiques sont transfrontalières de 
manière aléatoire ; où les habitudes économiques sont transfrontalières par opportunité, et enfin où les habitudes citoyennes sont transfrontalières par besoin, envie et opportunités mêlées. Gageons que dans ces territoires où les obstacles sont nombreux, faibles densités, complexités des systèmes institutionnels, mobilisation politique disparate, citoyens difficiles à toucher, les données et informations que nous avons traitées saurons convaincre les décideurs d'accompagner les coopérations de proximité et de leur donner les moyens de se développer pour une meilleure cohésion transfrontalière et le renforcement de ce que Roger Brunet nommait l'intégration spatiale (Brunet, 1994), qui consiste en la relation de lieux entre eux, nourrie par les liens de sorte que les parties de l'ensemble spatiale entretiennent plus de relations entre elles qu'avec l'extérieur. Une géographie assez complexe de la coopération se fait jour, plus fondée sur l'innovation, l'expérimentation et l'expertise (...), faisant ainsi apparaître une nouvelle territorialité (Claudot, 2016) que nous qualifierons ici de transfrontalière où les logiques de projets conduisent à l'émergence de nouvelles territorialités (Reitel, 2015). Enfin, il conviendrait d'interroger d'autres espaces frontaliers français, dans la relation qu'ils entretiennent avec le pays voisin dans la perspective du développement de petites coopérations de proximité. La méthodologie mise en place s'avère simple et facilement reproductible, et il serait intéressant de comparer si les dynamiques relevées dans notre enquête sont générales ou propres à l'espace étudié.

\section{BIBLIOGRAPHIE}

Brunet R., 1994, Les mots de la géographie, Reclus-la Documentation Française, Paris, 518 p.

Claudot R., 2016, Dynamique des coopérations transcommunales construites par les acteurs locaux des espaces ruraux wallons: Vers une nouvelle territorialité rurale? SPW Editions, Coll. Etudes et documents, Jambes, $170 \mathrm{p}$.

Conférence Transjurassienne (CTJ), 2016, Pour une nouvelle dynamique de l'Arc jurassien: Stratégie de coopération transfrontalière 2016-2020, 43 p.

Crevoisier O., Moine A., Signoret P., 2006, Impact de l'accord bilatéral relatif à la libre circulation des personnes sur la région transfrontalière du canton de Neuchâtel et du département du Doubs, Projet CANEDEDO, Université de Franche-Comté, Université de Neuchâtel, Rapport final, 17 p.

Dubois Y., Rérat P., 2012, «Vivre la frontière : les pratiques spatiales transfrontalières dans l'Arc jurassien franco-suisse », in Belgeo [En ligne].

Eder Sandtner S., Sandtner M., 2002, Une identité régionale transfrontalière ? La Regio TriRhena dans la prise de conscience de la population, in Revue Géographique de l'Est, vol. 42 / 1-2 | 2002

Fourny-Kober M-C., Crivelli R., 2003, Cette montagne que l'on partage. Frontière et montagne dans les coopérations transfrontalières de régions alpines, in Revue de Géographie Alpine, Volume $61, \mathrm{n}^{\circ} 3$, pp. 57-70.

Gadrey J., 2004, « L'utilité sociale des organisations de l'économie sociale et solidaire. Une mise en perspective sur la base de travaux récents », rapport pour la DIES-MiRe, février 2004. 
Gertsch K., Moine A., Rérat P., Ruefly E., Signoret P., Crevoisier O., 2012, La mobilité résidentielle transfrontalière et le fonctionnement du marché immobilier dans l'Arc jurassien franco-suisse, Projet MORETRADONE, Université de Franche-Comté, Université de Neuchâtel, Rapport final, 393 p.

Kramer H-L., 2004, « Le frontalier. Regard sociologique sur un être méconnu », in Revue européenne des sciences sociales, p. 199-218.

Le Lay Y-F., Rivière-Honnegger A., 2009, Expliquer l'inondation : la presse quotidienne régionale dans les Alpes et leur piedmont (1882-2005), in Géocarrefour, Vol. 84/4, pp. 259-270.

Leloup F., Moyart L., 2015, La région frontalière : vers quels nouveaux modes de développement et de gouvernance? in Après les frontières, avec la frontière. Nouvelles dynamiques transfrontalières en Europe, Ed. De l'Aube, La Tour d'Aigues, pp. 38-54.

Leresche J-P., Saez G., 1997, Identités territoriales et régimes politiques de la frontière, in Revue Pôle Sud, Volume 7, n¹, pp. 27-47.

Moine A., 2015, Les effets frontière dans l'Arc jurassien et leur impact sur les territoires ruraux frontaliers : Le cas du Val de Morteau, Chapitre 7, Revue Interrogations, Laboratoire C3S, Université de Franche-Comté, pp. 101-115.

Moine A., 2014, Effets frontière dans l'Arc jurassien, in Frontières, Lettres comtoises, Ed. ALAC, pp. 137-154.

Moine A., 2013, Quelles coopérations dans l'Arc jurassien franco-suisse, pour quel(s) aménagement(s) ?, in Les Cahiers Européens des Sciences Sociales, $\mathrm{n}^{\circ}$ spécial « Frontières et aménagement des territoires ", 5/2013, Ed. Balzac, Baixas, pp. 43-65.

Moine A., 2012, Les effets frontière dans l'Arc jurassien », Actes du colloque « L'Arc jurassien : frontière ou interface ? Fédération des Sociétés Savantes de Franche-Comté, Société d'Emulation du Doubs, Estimprim, Besançon, pp. 365-387.

Moulle F. 2013, La frontière et son double. Un modèle à partir de l'expérience européenne , in Belgéo, https://belgeo.revues.org/10620, consulté le 10/06/2016.

Paasi A., 1995, Territories, boundaries and consciousness : the changing geographies of the FinnishRussian Boundary, J. Wiley \& Sons, 353 p.

Paugam S., 2007, Repenser la solidarité : L'apport des sciences sociales, Presses universitaires de France, coll. «Quadrige Essais Débats », Paris, 980 p.

Reitel B, 2015, Les logiques de projet dans l'agglomération transfrontalière de Strasbourg-Kehl et de l'agglomération trinationale de Bâle : vers l'émergence de nouvelles territorialités ? in Après les frontières, avec la frontière. Nouvelles dynamiques transfrontalières en Europe, Ed. De l'Aube, La Tour d'Aigues, pp. 151-167

Rérat P., Moine A., Gertsch K., Signoret P., 2011, La mobilité résidentielle transfrontalière dans l'Arc jurassien franco-suisse, in Géo-Regard, « Mobilité et développement transfrontalier ", $\mathrm{n}^{\circ} 4$, Ed. Alphil, Presses Universitaires Suisses, Neuchâtel, pp. 153-168.

Rokkan S., 1970, Citizens elections parties: approaches to the comparative study processes of development, New York, Mac Key.

Segessemann A., 2015, Beyond Economic Base Theory: The Role of the Residential Economy, in Attracting Income to Swiss Regions, Regional Studies, ahead of print, pp. 1-16.

Simmel G., 1999, Etudes sur les formes de la socialisation, rééd. Paris, PUF.

Sohn C., 2014, Modelling Cross-Border Integration : The Role of Borders as a Resource, in Geopolitics, Vol. 19 , pp. 587-608. 
Sohn C., Walther Olivier, 2009, Métropolisation et intégration transfrontalière : le paradoxe luxembourgeois, in Espaces et sociétés 3/2009, Ed. ERES, (n 138), pp. 51-67.

\section{NOTES}

1. Voir les nombreux articles de presse et blogs sur cette question, notamment: Rue 89, «La haine des frontaliers, fonds de commerce de l'extrême droite genevoise " - http:// www.rue89lyon.fr/2014/09/25/geneve-la-haine-des-frontaliers-fonds-de-commerce-delextreme-droite/ ou La Tribune de Genève, «Le nombre de frontaliers a doublé en 10 ans »http://www.tdg.ch/suisse/Le-nombre-de-frontaliers-a-double-en-10-ans/story/31723339 ou encore Le Temps, « Frontaliers : des statistiques contre la chasse aux boucs émissaires » - https:// www.letemps.ch/suisse/2013/11/15/frontaliers-statistiques-contre-chasse-aux-boucs-emissaires 2. Michel Foucher précise dans un entretien accordé à «Jeune République» que «le transfrontalier est un laboratoire de création artistique, culturelle et d'échanges " - http:// www.geographie.ens.fr/IMG/file/Memoires_eleves/ENTRETIEN\%20AVEC\%20MICHEL\% 20FOUCHER_Sundar\%20Ramanadane.pdf

3. GEOSPECS 2012, ESPON TERCO 2013.

4. Aux 61 projets Interreg IV comptabilisé entre la Franche-Comté et les 4 cantons de JU, BE, NE et VD, nous retranchons 7 projets recensés lors de notre enquête et visibles localement.

5. Sur la base d'une estimation personnelle en lien avec les explications données par les porteurs de projets, les articles de la Presse Quotidienne Régionale.

6. Association suisse, mais dont les adhérents sont français et suisses, le président français et qui par convention du Conseil de l'Europe, est reconnue juridiquement en France, ceci permettant l'ouverture d'un compte bancaire et le dépôt de demandes de subvention auprès des collectivités françaises.

\section{RÉSUMÉS}

Un certain nombre de coopérations s'établissent plus ou moins spontanément de part et d'autre de la frontière franco-suisse dans l'Arc jurassien. En général de petite taille, visibles ou non aux échelles locales, elles présentent, selon nous, un grand intérêt dans le cadre de la mise en place d'un sentiment communautaire dans l'Arc jurassien. En effet, le devenir de cet espace transfrontalier doit s'ancrer dans le partage des valeurs des deux pays frontaliers afin d'endiguer les risques de repli identitaire. Dans sa stratégie de coopération transfrontalière 2016-2020, la Conférence Transjurassienne (CTJ) propose de promouvoir le sentiment d'une communauté de destin (CTJ, 2015), thématique également abordée par le Forum Transfrontalier (FT) dans son 5 ème cycle «Culture et identité (Forum Transfrontalier, 2016). Ainsi, si le sentiment identitaire partagé n'existe pas, force est de constater que les moyens pour l'initier passent par les collaborations de proximité. Rien de tel que des rencontres et des manifestations civiques, économiques, sociales et culturelles dynamiques et mobilisatrices pour entretenir un sentiment d'identité partagée. C'est sur la base de ce constat que le FT a lancé une vaste enquête à l'échelle de l'Arc jurassien, afin d'évaluer l'importance de ces coopérations, de comprendre de quelle manière elles naissaient, comment elles se développaient et sur quels moyens elles s'appuyaient. Menée durant un an à 
cheval sur 2015-2016, cette enquête a permis de mettre au jour près de 80 coopérations de proximité et d'en interroger plus d'une quarantaine. Les thématiques en jeu sont variées, les formes des coopérations également, tout autant d'ailleurs que les attentes des porteurs de projets dont les moyens sont finalement très réduits alors que les enjeux de cohésions sociale sont immenses... Nous rendons ainsi compte de l'inégale répartition spatiale de ces coopérations, de manières de faire souvent originale et nous pointons les chemins, les attentes et les besoins de ces porteurs de projet souvent discrets et pour le moins incontournables.

Beidseits der französisch-schweizerischen Grenze des Jurabogens haben sich mehr oder weniger spontan zahlreiche Kooperations-Initiativen gebildet. Sie sind im Allgemeinen klein, lokal und mehr oder weniger sichtbar, sind aber nach unserer Meinung für das Entstehen eines Zusammengehörigkeitsgefühls im Jurabogen von grosser Bedeutung. Die Entwicklung eines grenzüberschreitenden Territoriums muss sich im Erleben der gemeinsamen Werte verankern, um die Risiken für den Rückzug auf das bloss Eigene zu verhindern. Die Conférence Transjurassienne (CTJ) schlägt in ihrer Strategie für eine grenzüberschreitende Zusammenarbeit 2016-2020 vor, das Gemeinschaftsgefühl der Region (CTJ 2015) zu fördern, wie das thematisch auch das Forum Transfrontalier (FT) in seinem fünften Zyklus Kultur und Identität getan hat. Sofern das Gefühl einer gemeinsamen Identität nicht schon besteht, geht es also darum, die in nachbarschaftlicher Nähe operierenden Initiativen zu unterstützen. Es gibt dazu nichts Besseres als Zusammenkünfte und Veranstaltungen der Zivilgesellschaft, seien sie wirtschaftlicher, sozialer oder kultureller Natur, da sie mit ihrer Dynamik das Gefühl gemeinsamer Identität unterhalten und fördern.Auf der Basis dieser Feststellungen hat das FT im Jurabogen eine breitangelegte Untersuchung lanciert, um die Bedeutung dieser nachbarschaftlichen Kooperationen einzuschätzen und um zu verstehen, wie sie entstehen, sich entwickeln und auf was für Mittel sie sich stützen. Wir haben diese Befragung während eines ganzen Jahres, verteilt auf die Jahre 2015 und 2016, durchgeführt, und sie hat erlaubt, etwa 80 solche Organisationen aufzudecken und unter ihnen über vierzig zu befragen. Sie sind sowohl thematisch wie formal völlig verschiedener Natur, wie auch die Erwartungen der Verantwortlichen sehr unterschiedlich sind. Dabei steht in Sachen sozialer Kohäsion enorm viel auf dem Spiel. Wir berichten über die sehr ungleiche örtliche Verteilung diese Kooperationen, ihre oft sehr originelle Vorgehensweise, und wir weisen auf den Weg, die Erwartungen und Bedürfnisse ihrer Verantwortlichen hin, die oft sehr diskret und deswegen nicht minder wichtig sind.

Some of cooperations are established more or less spontaneously on part of the swiss border in Jura moutains. Generally small, more or less visble, they are interesting to build a sense of community above the border. Indeed, the future of this border territory must be based on shared values of the two countries to fight against identity stow. The strategy of the Conférence Transjurassienne (CTJ) about the border cooperation (2016-2020) is to propose the promotion of a common destiny (CTJ, 2015), the same subject which was treated by the Forum Transfrontalier (FT) during is fifth cycle "Culture and identity" (Forum Transfrontalier, 2016). In the fact, this sense of identity community is not shared, and the best way is to build proximity collaborations. Nothin's better than cultural or economics meetings, demonstrations of citizens, to share our respective identities. This is on the basis of this observation that the FT realized a broad survey in Jura region, to evalue the place of these "small" cooperations, to understand how they born, how they grow, how do they behave and finally what are their ressources. After identification of 80 cooperations we asked the half of them during one year. Their themes are varied, their expectations and their way to also and finally we find that their means are reduced while the challenges of this cooperation are immense... So, we show the unequal spatial distribution of these cooperations, some original ways to build some actions on both sides of the border and we show the expectations and needs of these project leaders, often subtle, yet essential. 
INDEX

Schlüsselwörter : Grenzüberschreitende Kooperation; Nachbarschaft; französischschweizerischer Jurabogen; Identität; Entwicklung; soziale Kohäsion

Keywords : border cooperation, Jura moutains, identity, development, social cohesion

Mots-clés : coopérations transfrontalières, proximité, Arc jurassien franco-suisse, identité, développement, cohésion sociale

\section{AUTEUR}

ALEXANDRE MOINE

Laboratoire Théma - UMR 6049 CNRS - Université de Bourgogne - Franche-Comté 